\title{
Oxidized phospholipids modify plasminogen and apolipoprotein(a): Implications for vascular disease ${ }^{*}$
}

\author{
Angelo M. Scanu ${ }^{1,2 \#}$, Diana M. Stafforini ${ }^{3}$, Celina Edelstein ${ }^{1}$ \\ ${ }^{1}$ Department of Medicine, University of Chicago, Chicago, USA \\ ${ }^{2}$ Department of Biochemistry and Molecular Biology, University of Chicago, Chicago, USA \\ ${ }^{3}$ Huntsman Cancer Institute, University of Utah, Salt Lake City, USA \\ Email: \#ascanu@medicine.bsd.uchicago.edu
}

Received 13 January 2012; revised 22 February 2012; accepted 8 March 2012

\begin{abstract}
Whereas the close structural homology between human plasminogen and apolipoprotein(a) has been known for a number of years only recent studies have revealed that both proteins carry linked oxidized phospholipids that may modify the function of these proteins. Future studies should provide a better understanding of oxidized phospholipid adducts and the role played by lipoprotein-associated phospholipase $A_{2}$ for which cleavage specificity has been established when these modified lipids are in a free form.
\end{abstract}

Keywords: Plasminogen; Apolipoprotein(a); Lipoprotein(a); Oxidized Phospholipids; Oxidized Phosphatidylcholines

\section{INTRODUCTION}

Early pioneering studies pointed at a high degree of homology between human plasminogen (Plg) and apolipoprotein(a) [apo(a)], a specific protein component of lipoprotein(a) [Lp(a] [1,2]. Lp(a) is an LDL-like particle in which apo(a) is covalently linked by a single disulfide bond to the apoB100 component of the complex. Despite their high degree of homology, there are important features that make Plg and apo(a) unique (Table 1). An essential difference is the occurrence in apo(a) of several identical kringle IV type 2 repeats that vary in number among individuals and that are key determinants of protein size. In addition, although not unequivocally established, the number of repeats appears to be inversely related to the plasma levels of $\mathrm{Lp}(\mathrm{a})$ [3]. Also of interest, the protease cleavage site expressed in Plg is absent in apo(a) because the Arg residue required for cleavage of Plg is replaced by Ser in apo(a) [1]. These seminal studies have stimulated considerable interest in the fields of

\footnotetext{
*Supported by NIH Grant HL63209 (A.M.S.) and NIH Grant HL35828-22 (D.M.S.).

${ }^{\#}$ Corresponding author.
}

atherosclerosis and thrombosis given that apo(a), a kringle-rich structure with thrombotic potential resulting from its structural similarity to Plg, is linked to pro-atherogenic cholesterol-rich lipoproteins [4,5].

Throughout the years, investigations on $\mathrm{Lp}(\mathrm{a}) / \mathrm{apo}(\mathrm{a})$ have been focused on epidemiological studies showing that high plasma levels of Lp(a) are associated with increased incidence of cardiovascular disease and stroke [4]. However, it is not clear whether $L p(a)$ actively participates in cardiovascular disease and, if so, what mechanisms account for potential effects [6]. A number of studies in an apparently unrelated line of investigations have pointed at the contribution of oxidized phospholipids (oxPL), and particularly oxidized phosphatidylcholines (oxPC), to the pathogenesis of cardiovascular disease [6,7]. Structural differences between oxidized and native PL affect their function and mode of interaction with cells, proteins, elements of the immune system, and role in the atherosclerotic process [8]. The fields of Lp(a) and oxPL pathophysiology merged with the seminal discovery that apo(a) is derivatized with oxPC under physiological conditions [9]. This finding suggested that the modification could account for pro-atherogenic properties of Lp(a), thus providing a molecular basis for its suspected contribution to disease pathogenesis. At the same time several studies have led to the proposal that Plg, like Lp(a), plays a role in cardiovascular disease [10]. The structural and functional similarities between apo(a) and Plg prompted investigations that culminated with the recent observation that purified Plg isolated from fresh and frozen plasma samples harbors covalently linked oxPCs, as judged by immunological and mass spectrometric analyses [11] hence the suggestion that linked oxPCs could account for, or exacerbate, the pathogenicity of Plg [11,12].

An important feature to emphasize is the specificity of protein derivatization with oxPL, as it is apparent that key domains expressed in a defined set of proteins confer susceptibility to modification. OxPCs are solely linked to 
Table1. Comparative properties of Plg and apo(a).

\begin{tabular}{ccc}
\hline & Plg & apo(a) \\
\hline Synthesis & Liver & Liver \\
Mass, kDa & 90 & $300-800$ \\
Number of kringles & 5 & $10-40$ \\
Protease domain & Active & Inactive \\
Zymogen & Yes & No \\
LDL linkage & No & Yes \\
\hline
\end{tabular}

the apo(a) protein component of $\mathrm{Lp}(\mathrm{a})$ and this linkage involves one or two specific lysine residues in kringle $\mathrm{V}$ $[9,13]$. Similarly, Plg harbors an average of two moles of oxPCs per mole of protein and the linkage likely occurs in kringle 5 (Figure 1). Importantly, this feature is typical of commercial and in-house preparations of human and mouse Plg from plasma or liver [11,12]. The relevance of this observation lies on the fact that mice do not produce apo(a) [13] and may thus transport the majority of oxPCs as Plg adducts. Plg is also the main carrier of oxPCs in rhesus monkeys (Edelstein, C. and Scanu, A.M., unpublished observation) since this species produces apo(a) that lacks the kringle $V$ domain [14] harboring the site for oxPC linkage and thus is devoid of oxPC [9]. It is important to note that under the physiological conditions examined, oxPC linkage is not a general property of all proteins in the plasma since T15 reactivity is absent in albumin the most abundant protein in the plasma and in two proteins of the blood coagulation system, FactorXII and prothrombin that contain one and two kringles, respectively [11].

A second issue is whether oxPL linkage preserves metabolic susceptibility and biological activities that characterize free oxPLs. The site and timing of derivatization can provide important clues regarding function. Notably, cultured HepG2 cells secrete Plg with linked oxPCs and all sources of Plg tested thus far are derivatized [11,12]. This suggests that under basal conditions adduct formation occurs during, or shortly following, biosynthesis in the liver. Moreover, linkage to proteins appears to protect oxPL from metabolism by lipoprotein-associated phospholipase $A_{2}\left(L p-P L A_{2}\right)$ [12] the major enzyme involved in oxPL metabolism [15]. Deletion of the gene encoding this enzyme does not alter basal levels of Plg adducts in the circulation [12]. These observations suggest that under physiological conditions both apo(a) and Plg may serve a beneficial role by facilitating export of pro-inflammatory oxPCs from the liver, transport in plasma in a metabolically inert form, and uneventful tissue disposal. This hypothesis is consistent with the lack of reported evidence of adduct-related pathology in the circulation under normal, physiological conditions.

In pathological settings, however, linkage of oxPL to
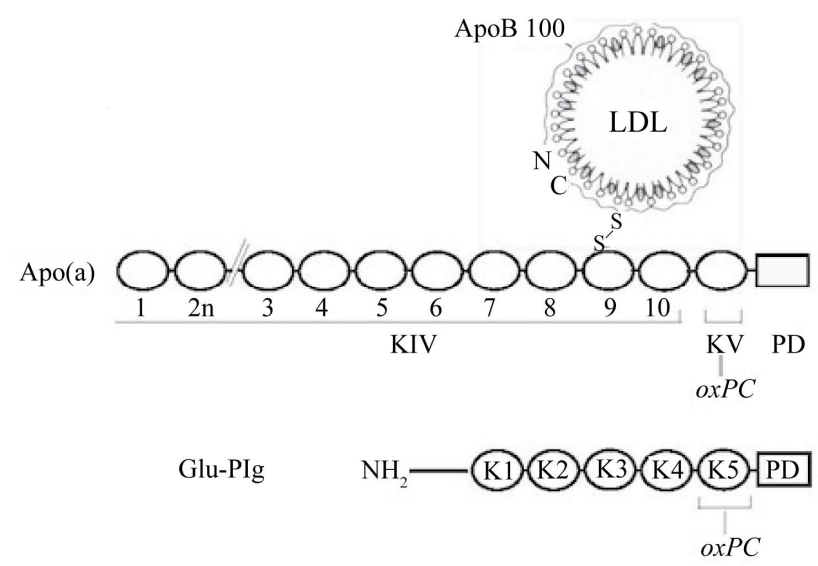

Figure 1. Schematic representation of the location of oxPCs in Lp(a) and Plg. Top. Lp(a). Shown is the LDL component wrapped around by apoB100 linked by a disulfide bridge to apo(a) that is made of 10 classes of kringle type IV followed by single copies of kringle $\mathrm{V}$ and the protease domain. The oxPCs are located in kringle V; none in the LDL particle [9]. Bottom. Plg showing the $\mathrm{NH}_{2}$ terminal peptide, followed by kringles 1 to 5 , and the protease domain. The oxPCs are located in kringle 5.

Plg and apo(a) may have other functional consequences. Studies in cultured human macrophages have shown that the presence of linked oxPC imparts pro-inflammatory properties to apo(a) as these preparations stimulate the production of IL-8 [9]. Interestingly, studies in arte- rial endothelial cells have shown that Plg mediated IL-8 production is enhanced when Plg is proteolytically cleaved by elastase to favor the exposure of oxPCs to the action

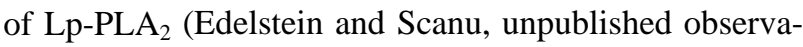
tions). This response likely represents the effect of lysoPCs released by the action of exogenous Lp-PLA 2 . In this regard, a recent study has shown that an inhibitor of Lp-PLA $A_{2}$, which presumably decreases the content of lyso-PC in atherosclerotic plaques, diminishes plaque formation and inflammation in two models of atherosclerosis $[16,17]$. However, it remains to be established whether this effect represents inhibition of endogenous Lp$\mathrm{PLA}_{2}$-mediated hydrolysis of protein-linked or free oxPC in atherosclerotic plaques.

In summary, we have outlined several key features and possible functional consequences associated with derivatization of apo(a) and Plg with oxPLs. First, is the observation that Plg, a key zymogen in the fibrinolytic system, and apo(a) harbor covalently linked oxPCs. Second, is the notion that derivatization occurs in two genetically related proteins and appears to require expression of kringle domains. Third, is the unexpected observation that linkage is likely occurring during protein biosynthesis and under normal, unstimulated conditions. Fourth, is the finding that protein linked oxPCs are resistant to hydrolysis by Lp-PLA ${ }_{2}$ and the possibility that linkage may provide a mechanism for oxPL safe transport. Fifth, is 
the potential of protein-linked oxPC to impart proatherogenic and pro-inflammatory functions in pathological settings, mainly through Lp-PLA ${ }_{2}$-mediated release of lyso-PC. In consequence, very careful attention should be directed at Lp-PLA $A_{2}$ given its specificity to cleave oxPCs. From a cardiovascular standpoint, it would be important to assess whether Lp-PLA $A_{2}$ can locally release lyso-PC from protein-linked oxPCs even though it appears not to recognize these adducts in the circulation. In addition, it will be essential to take into consideration recent work showing that Lp-PLA $A_{2}$ can have multifaceted consequences depending on the structural nature of the oxPL metabolized [18]. Overall, the biological role of oxPL adducts remains to be established. We believe that Plg and apo(a) provide an excellent paradigm for defining this role both under physiological and pathological conditions.

\section{REFERENCES}

[1] McLean, J.W., Tomlinson, J.E., Kuang, W., Eaton, D.L., Chen, E.Y., Fless, G.M., Scanu, A.M. and Lawn, R.M. (1987) cDNA sequence of human apolipoprotein(a) is homologous to plasminogen. Nature, 330, 132-137. doi:10.1038/330132a0

[2] Scanu, A.M. and Fless, G.M. (1990) Lipoprotein(a): Heterogeneity and biological relevance. Journal of Clinical Investigation, 85, 1709-1715. doi:10.1172/JCI114625

[3] Hobbs, H.H. and White, A.L. (1999) Lipoprotein(a): Intrigues and insights. Current Opinion in Lipidology, 10, 225-236. doi:10.1097/00041433-199906000-00005

[4] Scanu, A.M. (1992) Lipoprotein(a) —Its inheritance and molecular basis of its atherothrombotic role. Molecular and Cellular Biochemistry, 113, 127-131. doi:10.1007/BF00231532

[5] Scanu, A.M. (2003) Lipoprotein(a) and the atherothrombotic process: Mechanistic insights and clinical implications. Current Atherosclerosis Reports, 5, 106-113. doi:10.1007/s11883-003-0081-3

[6] Tsimikas, S. and Witztum, J.L. (2008) The role of oxidized phospholipids in mediating lipoprotein(a) atherogenicity. Current Opinion in Lipidology, 19, 369-377. doi:10.1097/MOL.0b013e328308b622

[7] Philips, B. and Scanu, A.M. (2008) Viewing the cardiovascular pathogenicity of $\mathrm{Lp}(\mathrm{a})$ from the pro-inflammatory side. Vascular Disease Prevention, 5, 150-155.

[8] Berliner, J.A., Leitinger, N. and Tsimikas, S. (2009) The role of oxidized phospholipids in atherosclerosis. Journal of Lipid Research, 50, S207-S212. doi:10.1194/jlr.R800074-JLR200
[9] Edelstein, C., Pfaffinger, D., Hinman, J., Miller, E., Lipkind, G., Tsimikas, S., Bergmark, C., Getz, G.S., Witztum, J.L. and Scanu, A.M. (2003) Lysine-phosphatidylcholine adducts in kringle $\mathrm{V}$ impart unique immunological and potential pro-inflammatory properties to human apolipoprotein(a). Journal of Biological Chemistry, 26, 52841528447. doi:10.1074/jbc.M310425200

[10] Plow, E.F. and Hoover-Plow, J. (2004) The functions of plasminogen in cardiovascular disease. Trends in Cardiovascular Medicine, 14, 180-186. doi:10.1016/j.tcm.2004.04.001

[11] Edelstein, C., Pfaffinger, D., Yang, M., Hill, J.S. and Scanu, A.M. (2010) Naturally occurring human plasminogen, like genetically related apolipoprotein(a), contains oxidized phosphatidylcholine adducts. Biochimica et Biophysica Acta, 1801, 738-745.

[12] Edelstein, C., Pfaffinger, D., Reichert, E.C., Stafforini, D.M. and Scanu, A.M. (2010) Mouse plasminogen has oxidized phosphatidylcholine adducts that are not metabolized by lipoprotein-associated phospholipase A2 under basal conditions. International Journal of Molecular Science, 11, 5339-5347.

[13] Edelstein, C., Philips, B., Pfaffinger, D. and Scanu, A.M. (2009) The oxidized phospholipids linked to human apolipoprotein(a) do not derive from circulating lowdensity lipoproteins and are probably of cellular origin. FASEB Journal, 23, 950-956. doi:10.1096/fj.08-122002

[14] Tomlinson, J., McLean, J. and Lawn, R. (1989) Rhesus monkey apolipoprotein(a). Journal of Biological Chemistry, 264, 5957-5965.

[15] Stafforini, D.M. (2009) Biology of platelet-activating factor acetylhydrolase (PAF-AH), lipoprotein associated phospholipase $\mathrm{A}_{2}$. Cardiovascular Drugs Therapeutics, 23, 73-83. doi:10.1007/s10557-008-6133-8

[16] Hu, M.M., Zhang, J., Wang, W.Y., Wu, Y.L., Ma, W.H. and Chen, W.P. (2011) The inhibition of lipoprotein-associated phos-pho-lipase A2 exerts beneficial effects against athero-sclerosis in LDLR-deficient mice. Acta Pharmacologica Sinica, 32, 1253-1258.

[17] Wang, W.Y., Zhang, J., Wu, W.Y., Li, J., Ma, Y.L., Chen, W.H., Yan, H., Wang, K., Xu, W.W., Shen, J.H. and Wang, Y.P. (2011) Inhibition of lipoprotein-associated phospholipase A2 ameliorates inflammation and decreases atherosclerotic plaque formation in apoE-defocient mice. PLoS One, 6, e234-e235.

[18] Vladykovskaya, E., Ozhegov, E., Hoetker, J.D., Xie, Z., Ahmed, Y., Suttles, J., Srivastava, S., Bhatnagar, A. and Barski, O.A. (2011) Reductive metabolism increases the proinflammatory activity of aldehyde phospholipids. Journal of Lipid Research, 52, 2209-2225. doi:10.1194/jlr.M013854 